\title{
A Tired Light/Contracting Universe Model from the Union2.1 Supernovae Data
}

\author{
John Glover \\ Chemical Engineering Department, Loughborough University, Loughborough, UK \\ Email: john.glover23@ntlworld.com
}

How to cite this paper: Glover, J. (2017) A Tired Light/Contracting Universe Model from the Union2.1 Supernovae Data. Journal of Modern Physics, 8, 147-155. https://doi.org/10.4236/jmp.2017.82013

Received: January 2, 2017

Accepted: February 10, 2017

Published: February 13, 2017

Copyright $\odot 2017$ by author and Scientific Research Publishing Inc. This work is licensed under the Creative Commons Attribution International License (CC BY 4.0).

http://creativecommons.org/licenses/by/4.0/

\begin{abstract}
A tired light/contracting universe (TLCU) model is shown to be an excellent fit to the redshift/distance modulus data for the 580 supernovae 1a in the Union2.1 compilation. The data reveal that the Milky Way is in a static region with a radius of about $450 \mathrm{Mpc}$. Beyond the static region the universe is contracting with a space velocity which is linearly proportional to distance over the whole range of the data $\left(k=-7.6 \pm 2.3 \mathrm{~km} \cdot \mathrm{s}^{-1} \cdot \mathrm{Mpc}^{-1}\right)$. The other constant of the model is the Hubble constant for which a value of $H=69.51 \pm 0.86 \mathrm{~km} \cdot \mathrm{s}^{-1} \cdot \mathrm{Mpc}^{-1}$ is obtained. The fit of the TLCU model to the Union2.1 data is at least as good as the fit of the two constant $\Lambda$ CDM model to the same data. A formula for photon travel distance is derived and an experiment for the possible detection of the tired light process is proposed.
\end{abstract}

\section{Keywords}

Cosmology, Observations, Theory

\section{Introduction}

Theory [1] predicts that the universe is either expanding or contracting with a space velocity which is linearly proportional to distance. An expansion may continue for ever or it may halt and then contract giving rise to the possibility of a "periodic world".

When it was observed that the redshift and distance for galaxies beyond the Local Group had a linear relationship [2] the expanding universe theory became established. Observations of supernovae 1a show that at higher redshifts [3], [4] the distances measured are greater than expected by the expanding universe theory and this is interpreted as an accelerating expansion for which a new force called "dark energy" is proposed.

The tired light theory [5] is an alternative explanation for the systematic 
redshift. The viewpoint on the supernovae 1a observations from the tired light theory is that for a given distance the observed redshift is less than expected as a result of the blueshift of a contracting universe. Hence it is suggested that the new physics required by the supernovae la observations may possibly be the old idea of tired light instead of the new idea of dark energy.

A tired light/contracting universe (TLCU) model is developed here using the Union2.1 supernovae 1a data [6] and compared to the $\Lambda$ CDM model. The TLCU model uses photon travel distance for which a formula is derived. A possible mechanism for the tired light effect is discussed and an experiment to test this mechanism is proposed.

\section{The TLCU Model}

The TLCU model is built on the idea [7] that the observed systematic redshift ( $z$ ) has two components

$$
z=z_{t l}+z_{c}
$$

where $z_{t l}$ is the result of an energy loss process and $z_{c}$ is the result of space contraction. Assuming that photons lose energy by a first order rate process [8] the tired light component is given by

$$
z_{t l}=\exp \left(\frac{H \times d}{c}\right)-1
$$

where $d$ is the photon travel distance, $H$ is the Hubble constant ${ }^{1}$ and $c$ is the speed of light. The distance between the emitter and observer at the moment the photon is emitted $\left(d_{0}\right)$ is here called the "initial distance" and $d \approx d_{0}$ for low values of $d_{0}$. The exact relationship between $d_{0}$ and $d$ is considered later. Assuming a flat (i.e. Euclidean) universe the initial distance is related [9] to the luminosity distance $(D)$ by

$$
d_{0}=\frac{D}{(1+z)}
$$

The luminosity distance $(D)$ is obtained from the distance modulus $(\mathrm{dm})$ by the standard relationship.

$$
\log _{10} D=\frac{d m+5}{5}
$$

The distance modulus $(d m)$ is defined as $d m=m-M$, where $m$ is the observed apparent magnitude of an object and $M$ is it's absolute magnitude. For supernovae 1a " $m$ " is the peak observed apparent magnitude (with appropriate corrections).

\subsection{Preliminary Calculations}

Initially $\mathrm{z}_{c}$ was calculated from the redshift and distance modulus data for

${ }^{1}$ The Hubble constant used in the TLCU model is a constant of nature and assumed to be independent of time and space. The usual units used for the Hubble constant are $\mathrm{km} \cdot \mathrm{s}^{-1} \cdot \mathrm{Mpc}^{-1}$ although the SI unit is $\mathrm{s}^{-1}$, which is characteristic of a first order rate process.

$\left(70 \mathrm{~km} \cdot \mathrm{s}^{-1} \cdot \mathrm{Mpc}^{-1} \equiv 2.27 \times 10^{-18} \mathrm{~s}^{-1}\right.$ ). 
each of the $580 \mathrm{SN}$ 1a in the Union2.1 compilation using Equations (1) to (4) and with the assumptions that $d=d_{0}$ and $H=70$. The results of the calculations are shown in Figure 1.

The 580 values of $Z_{c}$ consist of 158 positive values (redshifts) and 422 negative values (blueshifts). In a static universe $z_{c}$ would consist of an equal number of redshifts/blueshifts and the 158/422 split rejects a static universe $\left(p<10^{-10}\right)$. Since $z_{c}$ is essentially negative the initial assumption of a contracting universe is confirmed.

Although the overall picture is of a contracting universe the local situation is different. There are 176 supernovae with $0.015<z<0.101$ for which the $Z_{c}$ values show 78 redshifts and 98 blueshifts which is consistent $(p=0.13)$ with an even split. So the next conclusion is that the Milky Way is situated in a static region of about $450 \mathrm{Mpc}$ radius.

\subsection{The Final Model}

In a gravitationally bound region of space the force of Newtonian gravity is greater than the cosmic force of expansion/contraction and although the region as a whole will take part in the universal cosmic expansion/contraction the effect of cosmic expansion/contraction cannot be measured within the region. It is now assumed that the static region extending to about $450 \mathrm{Mpc}$ around the Milky Way is gravitationally bound. For the purpose of the model it is assumed that the Milky Way is located at the center of a static sphere with a radius of 450 $\mathrm{Mpc}$. In order to be consistent with the observations it is also assumed that the cosmic contraction starts at the edge of the static sphere. For a cosmic contraction the velocity of contraction is proportional to distance, so that

$$
z_{c} \times c=k \times\left(d_{0}-450\right)
$$

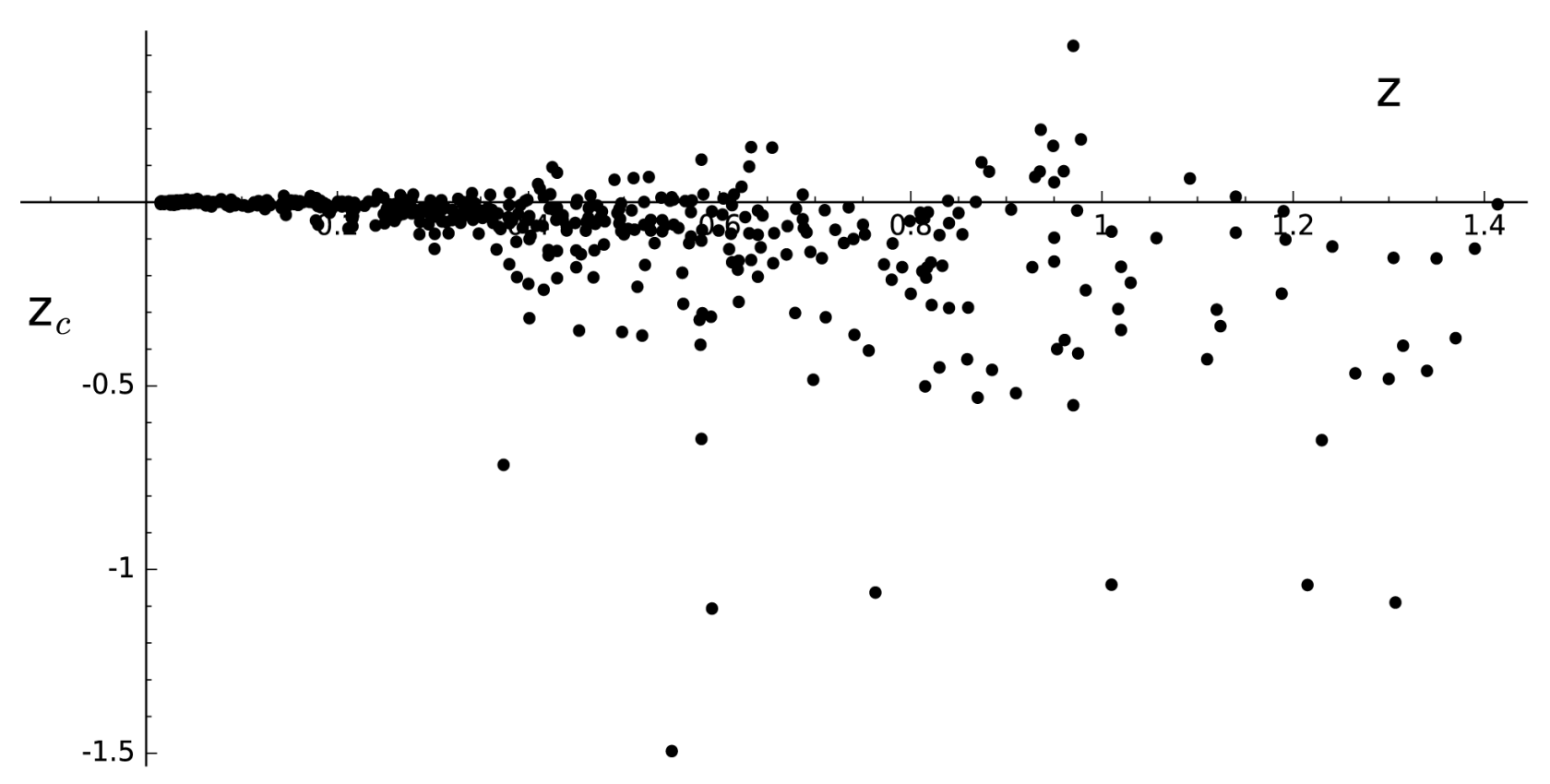

Figure 1. 580 values of $Z_{c}$ from the Union2.1 supernovae data with $H=70$. 
where $k\left(\mathrm{~km} \cdot \mathrm{s}^{-1} \cdot \mathrm{Mpc}^{-1}\right)$ is the constant for cosmic contraction. Within the static region $d=d_{0}$, but, beyond the static region it is necessary to allow for the contraction that occurs during the photon travel time. The relationship between $d_{0}, k$ and $d$ is derived in Appendix 1, from which, Equation (11) re-arranged as

$$
d=d_{o} \times \exp \left(\frac{k \times d}{c}\right)
$$

is more convenient for finding $d$ from $d_{o}$ and $k$ by repeated substitution. Equation (6) was used to calculate that part of the photon travel distance which is beyond the static region. The values of $H$ and $k$ for the TLCU model were found by fitting the model to the Union2.1 data. The sum of the weighted squares of the the $\mathrm{dm}$ residuals $(S)$ is given by

$$
S=\sum_{1}^{n}\left(d m_{\text {observed }}-d m_{\text {model }}\right)_{i}^{2} \times w_{i}
$$

where the weighting factor $(w)$ is proportional to the inverse square of the estimated error in $\mathrm{dm}$. For the Union2.1 data $(n=580)$, the values of the constants which minimize $S$ are $H=69.2 \pm 5.0 \mathrm{~km} \cdot \mathrm{s}^{-1} \cdot \mathrm{Mpc}^{-1}$ and $k=-7.1 \pm 10.3 \mathrm{~km} \cdot \mathrm{s}^{-1} \cdot \mathrm{Mpc}^{-1}$. The rms weighted residual $d m=0.170 \mathrm{mag}$. while the rms un-weighted residual $d m=0.226 \mathrm{mag}$.

Figure 1 shows a large scatter in $z_{c}$ so the data were binned in order to make the relationship between velocity of contraction and distance more precise. The $580 \mathrm{SN} \mathrm{1a}$ were sorted into $z$ order and divided into 29 bins each containing 20 SN 1a. The weighted average redshift and distance modulus were calculated for each bin (see Table 1). The procedure for finding $H$ and $k$ was repeated using the bin averages of redshift and distance modulus and gave $H=69.51 \pm 0.86$ and $k=-7.6 \pm 2.3 \mathrm{~km} \cdot \mathrm{s}^{-1} \cdot \mathrm{Mpc}^{-1}$ with the rms of the residual $\mathrm{dm}=0.030 \mathrm{mag}$. The values of the contraction velocity calculated from the binned data using using $H=69.5 \mathrm{~km} \cdot \mathrm{s}^{-1} \cdot \mathrm{Mpc}^{-1}$ and $k=-7.6 \mathrm{~km} \cdot \mathrm{s}^{-1} \cdot \mathrm{Mpc}^{-1}$ are shown in Figure 2. It is seen that the contraction velocity has an approximate linear relationship with distance beyond the static region over the whole range of the Union2.1 data. Hence the assumptions on which the model is based are consistent with the observed data. Figure 2 also shows a hint of periodicity in the velocity/distance relationship.

\section{Comparison of TLCU and $\Lambda$ CDM Models}

In the two constant $\Lambda \mathrm{CDM}$ model the initial distance (from Equation (13) of ref. $[10])$ is

$$
d_{0}=c / H_{0} \times \int_{o}^{z}\left[(1+z)^{3} \times \Omega_{M}+1-\Omega_{M}\right]^{-0.5} \mathrm{~d} z
$$

In order to compare the models on the same basis the constants for the $\Lambda \mathrm{CDM}$ model were found by fitting Equation (8) to the Union2.1 data using the best fit criterium (Equation (6)). This gave $H_{0}=70.0 \pm 5.4 \mathrm{~km} \cdot \mathrm{s}^{-1} \cdot \mathrm{Mpc}^{-1}$ and $\Omega_{M}=0.278$ with a rms weighted residual $d m=0.170$ while the rms un- 
Table 1. The bin Hubble constants for the TLCU and $\Lambda$ CDM models.

\begin{tabular}{|c|c|c|c|c|c|c|c|c|c|}
\hline \multirow{2}{*}{$Z$} & \multirow{2}{*}{$d m$} & \multirow{2}{*}{$\begin{array}{c}d_{o} \\
\mathrm{Mpc}\end{array}$} & \multirow{2}{*}{$\begin{array}{c}d \\
\mathrm{Mpc}\end{array}$} & \multirow{2}{*}{$z_{c}$} & \multirow{2}{*}{$Z_{t l}$} & \multicolumn{2}{|c|}{ TLCU } & \multicolumn{2}{|c|}{$\Lambda \mathrm{CDM}$} \\
\hline & & & & & & $H$ & resid. & $H_{o}$ & resid. \\
\hline (1) & (2) & (3) & (4) & (5) & (6) & (7) & (8) & (9) & (10) \\
\hline 0.0157 & 34.158 & 67 & 67 & 0 & 0.0157 & 69.99 & -0.79 & 70.31 & -0.31 \\
\hline 0.0192 & 34.589 & 81 & 81 & 0 & 0.0192 & 70.33 & -1.13 & 70.71 & -0.71 \\
\hline 0.0231 & 35.033 & 99 & 99 & 0 & 0.0231 & 69.05 & 0.15 & 69.51 & 0.49 \\
\hline 0.0260 & 35.294 & 112 & 112 & 0 & 0.0260 & 68.99 & 0.21 & 69.51 & 0.49 \\
\hline 0.0304 & 35.659 & 131 & 131 & 0 & 0.0304 & 68.34 & 0.86 & 68.93 & 1.07 \\
\hline 0.0338 & 35.862 & 144 & 144 & 0 & 0.0338 & 69.21 & -0.01 & 69.88 & 0.12 \\
\hline 0.0437 & 36.409 & 183 & 183 & 0 & 0.0437 & 69.90 & -0.70 & 70.76 & -0.76 \\
\hline 0.0597 & 37.109 & 249 & 249 & 0 & 0.0597 & 69.72 & -0.52 & 70.87 & -0.87 \\
\hline 0.0894 & 38.033 & 371 & 371 & 0 & 0.0894 & 69.15 & 0.05 & 70.82 & -0.82 \\
\hline 0.1287 & 38.894 & 532 & 532 & -0.0020 & 0.1307 & 69.18 & 0.02 & 70.49 & -0.49 \\
\hline 0.1632 & 39.444 & 665 & 664 & -0.0051 & 0.1683 & 70.20 & -1.00 & 70.94 & -0.94 \\
\hline 0.1976 & 39.935 & 810 & 807 & -0.0085 & 0.2061 & 69.58 & -0.38 & 69.96 & 0.04 \\
\hline 0.2352 & 40.378 & 964 & 957 & -0.0122 & 0.2474 & 69.22 & -0.02 & 69.44 & 0.56 \\
\hline 0.2641 & 40.646 & 1065 & 1056 & -0.0146 & 0.2786 & 69.76 & -0.56 & 70.04 & -0.04 \\
\hline 0.2914 & 40.899 & 1171 & 1159 & -0.0171 & 0.3085 & 69.52 & -0.32 & 69.80 & 0.20 \\
\hline 0.3243 & 41.135 & 1274 & 1258 & -0.0195 & 0.3438 & 70.42 & -1.22 & 70.88 & -0.88 \\
\hline 0.3554 & 41.416 & 1416 & 1395 & -0.0229 & 0.3782 & 68.96 & 0.24 & 69.33 & 0.67 \\
\hline 0.3897 & 41.671 & 1553 & 1526 & -0.0261 & 0.4159 & 68.33 & 0.87 & 68.73 & 1.27 \\
\hline 0.4221 & 41.862 & 1658 & 1625 & -0.0286 & 0.4507 & 68.66 & 0.54 & 69.19 & 0.81 \\
\hline 0.4538 & 42.076 & 1789 & 1749 & -0.0317 & 0.4855 & 67.85 & 1.35 & 68.37 & 1.63 \\
\hline 0.5017 & 42.279 & 1902 & 1855 & -0.0344 & 0.5361 & 69.38 & -0.18 & 70.24 & -0.24 \\
\hline 0.5427 & 42.440 & 1994 & 1941 & -0.0366 & 0.5793 & 70.60 & -1.40 & 71.73 & -1.73 \\
\hline 0.5895 & 42.712 & 2194 & 2126 & -0.0413 & 0.6308 & 68.97 & 0.23 & 69.98 & 0.02 \\
\hline 0.6302 & 42.860 & 2289 & 2214 & -0.0436 & 0.6737 & 69.74 & -0.54 & 70.94 & -0.94 \\
\hline 0.7080 & 43.180 & 2533 & 2437 & -0.0493 & 0.7573 & 69.36 & -0.16 & 70.62 & -0.62 \\
\hline 0.8011 & 43.624 & 2946 & 2810 & -0.0591 & 0.8602 & 66.21 & 2.99 & 67.06 & 2.94 \\
\hline 0.8799 & 43.722 & 2953 & 2817 & -0.0593 & 0.9391 & 70.49 & -1.29 & 72.01 & -2.01 \\
\hline 1.0060 & 44.130 & 3340 & 3160 & -0.0684 & 1.0745 & 69.22 & -0.02 & 70.49 & -0.49 \\
\hline 1.2559 & 44.763 & 3974 & 3712 & -0.0835 & 1.3394 & 68.64 & 0.56 & 69.45 & 0.55 \\
\hline
\end{tabular}

(1) weighted bin average redshift; (2) weighted bin average distance modulus; (3) initial proper distance-Equations (3) \& (4); (4) photon travel distance Equation (6); (5) cosmic blueshift If $d_{o}<450$ then $z_{c}=$ 0 else $z_{c}=(-7.6 / \mathrm{c}) \times\left(d_{o}-450\right) ;(6)$ tired light redshift $z_{t l}=z-z_{\dot{\delta}}(7) \mathrm{H}=(\mathrm{c} / \mathrm{d}) \times \ln \left(1+z_{t}\right)$; (8) resid $=69.2-H$; (9) Equation (8) $\Omega_{\mathrm{M}}=0.278$; (10) resid $=70.0-H_{o}$.

weighted $\mathrm{dm}$ residual $=0.266$. These residuals are identical to the residuals from the TLCU model. There is also a close correlation between the un-weightd dm residuals for the $\Lambda \mathrm{CDM}$ model and those for the TLCU model as seen in Figure 3. Thus the fit of the TLCU model to the Union2.1 data is nearly identical to the fit of the $\Lambda \mathrm{CDM}$ model to the same data. 


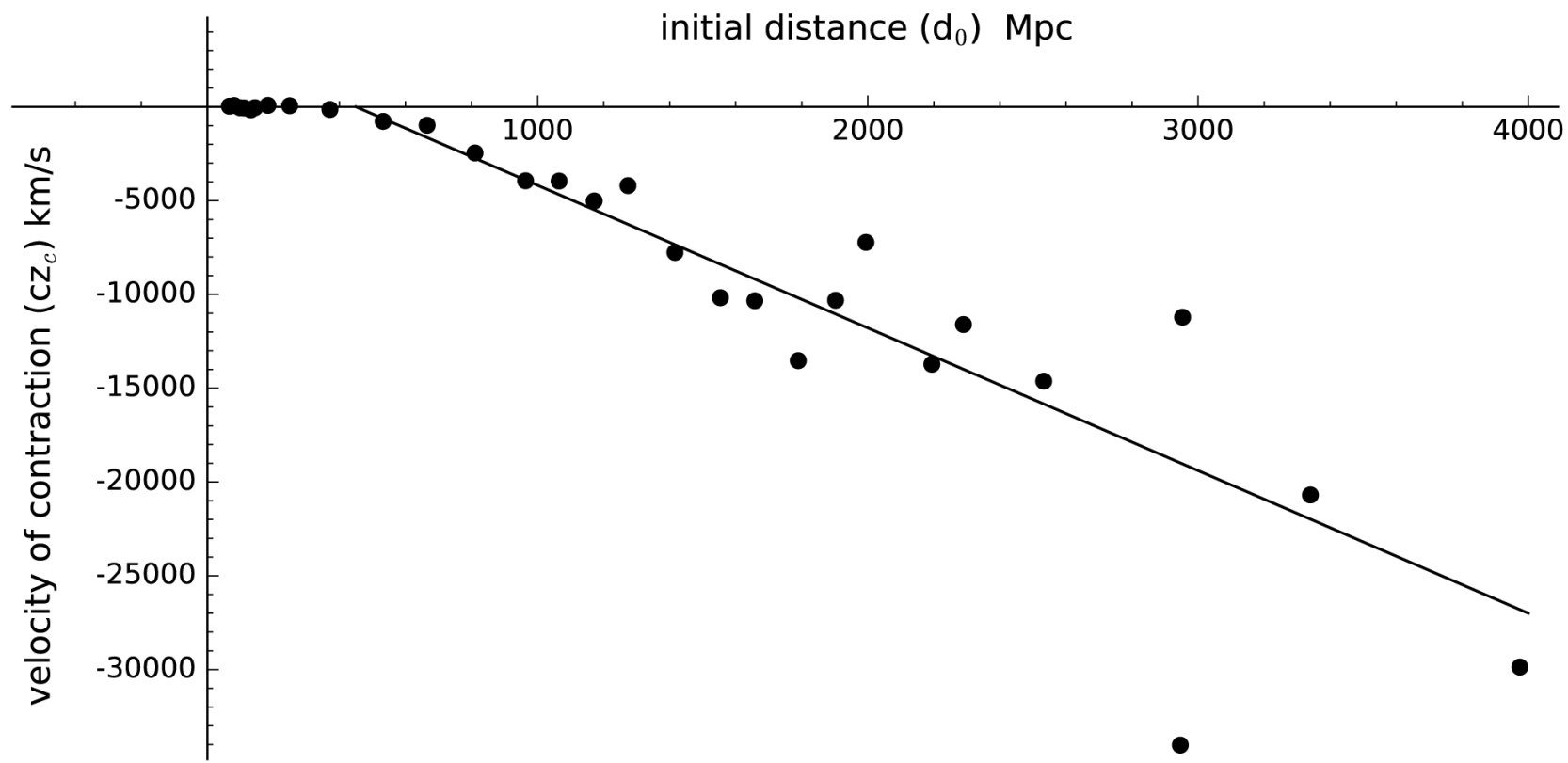

Figure 2. $z_{c}$ from the binned Union2.1 data (see text) for the TLCU model with $H=69.5 \mathrm{~km} \cdot \mathrm{s}^{-1} \cdot \mathrm{Mpc}^{-1}$ and $k=-7.6 \mathrm{~km} \cdot \mathrm{s}^{-1} \cdot \mathrm{Mpc}^{-1}$.

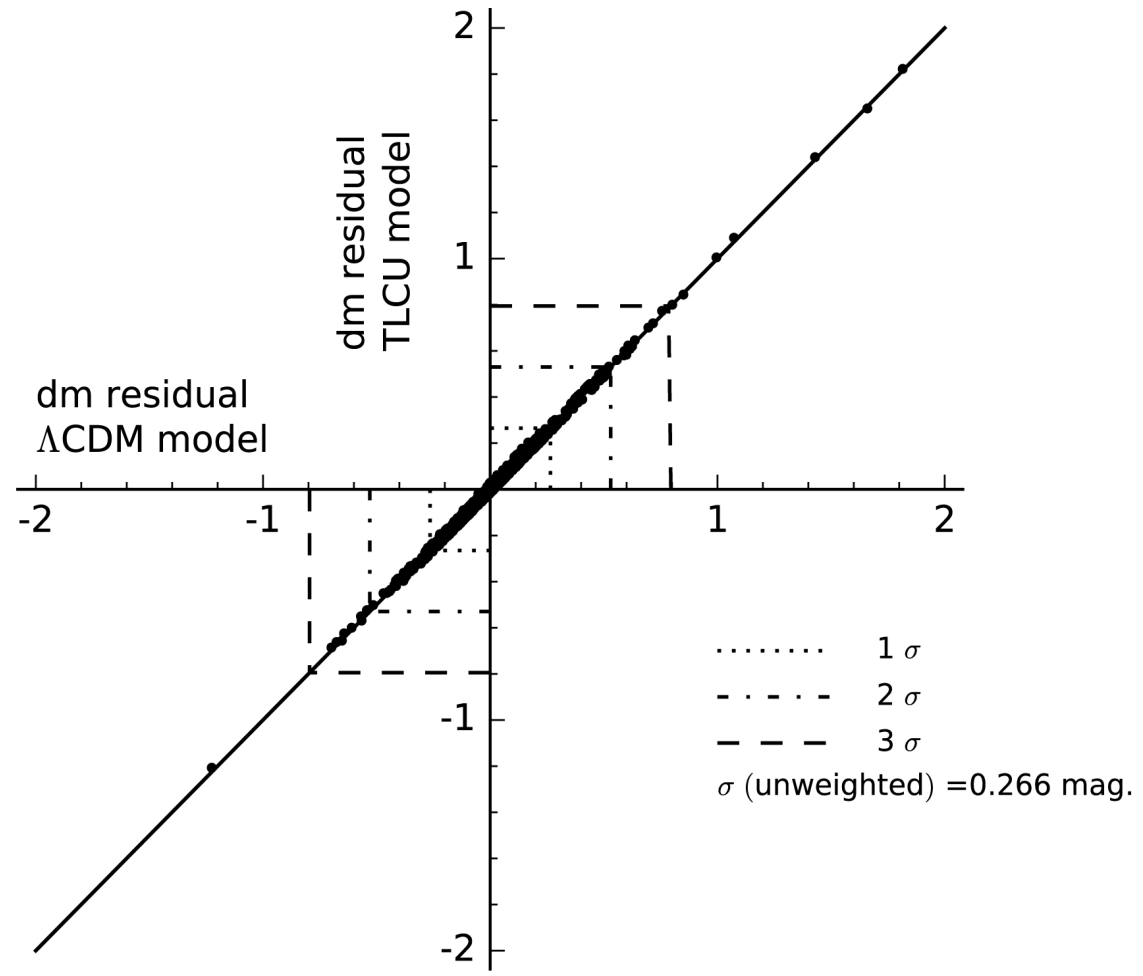

Figure 3. $d m$ residuals for $\Lambda \mathrm{CDM}$ and TLCU models using Union2.1 supernovae data.

Fitting the $\Lambda \mathrm{CDM}$ model to the binned data $(n=29)$ gives $H_{0}=70.09 \pm 1.01 \mathrm{~km} \cdot \mathrm{s}^{-1} \cdot \mathrm{Mpc}^{-1}, \Omega_{M}=0.274$ with a rms residual $\mathrm{dm}$ of 0.031 mag. The uncertainties in the Hubble constant and for the calculated $\mathrm{dm}$ are slightly greater than those for the TLCU model. The $\mathrm{dm}$ residuals for the binned 
data for both models are shown in Figure 4.

The hint of periodicity shown in Figure 2 is repeated in Figure 4 and not only for the TLCU model but also for the $\Lambda$ CDM model.

Another method of comparing the models is to fix the minor constants and then to calculate the value of the Hubble constant for each bin of the binned data. This calculation (with $k=-7.6 \mathrm{~km} \cdot \mathrm{s}^{-1} \cdot \mathrm{Mpc}^{-1}$ for the TLCU model and $\Omega_{M}=0.274$ for the $\Lambda \mathrm{CDM}$ model) gives $H=69.51 \pm 0.86 \mathrm{~km} \cdot \mathrm{s}^{-1} \cdot \mathrm{Mpc}^{-1}$ for the TLCU model and $H_{0}=70.10 \pm 1.01 \mathrm{~km} \cdot \mathrm{s}^{-1} \cdot \mathrm{Mpc}^{-1}$ for the $\Lambda$ CDM model. The results of these calculations are presented in Table 1 . The values of the uncertainty in the Hubble constant show that the TLCU model is a better fit to the binned data than is the $\Lambda$ CDM model.

\section{Discussion}

Although the TLCU model only shows a contracting universe it is reasonable to assume that there was a prior expansion which would be consistent with the "periodic world" predicted by Friedman [1]. In this case the linear contraction revealed by the model can be expected to reverse at higher redshifts and eventually show the expanding phase. The periodicity hinted at in Figure 2 and Figure 4 may possibly be harmonics of the fundamental period. More accurate observations at higher redshifts are needed to reveal the truth.

The reality of the contracting universe depends, of course, on the reality of the tired light effect and although the TLCU model is an excellent fit to the observed data such a fit is no guarantee of the reality of the assumptions on which the model is based. It is also claimed [11] that time dilation falsifies the tired light theory although the assumption that the thirteen high redshift supernovae used are not subject to a Malmquist type bias may not possibly be the case. Nevertheless independent evidence for the tired light effect is essential. A possible

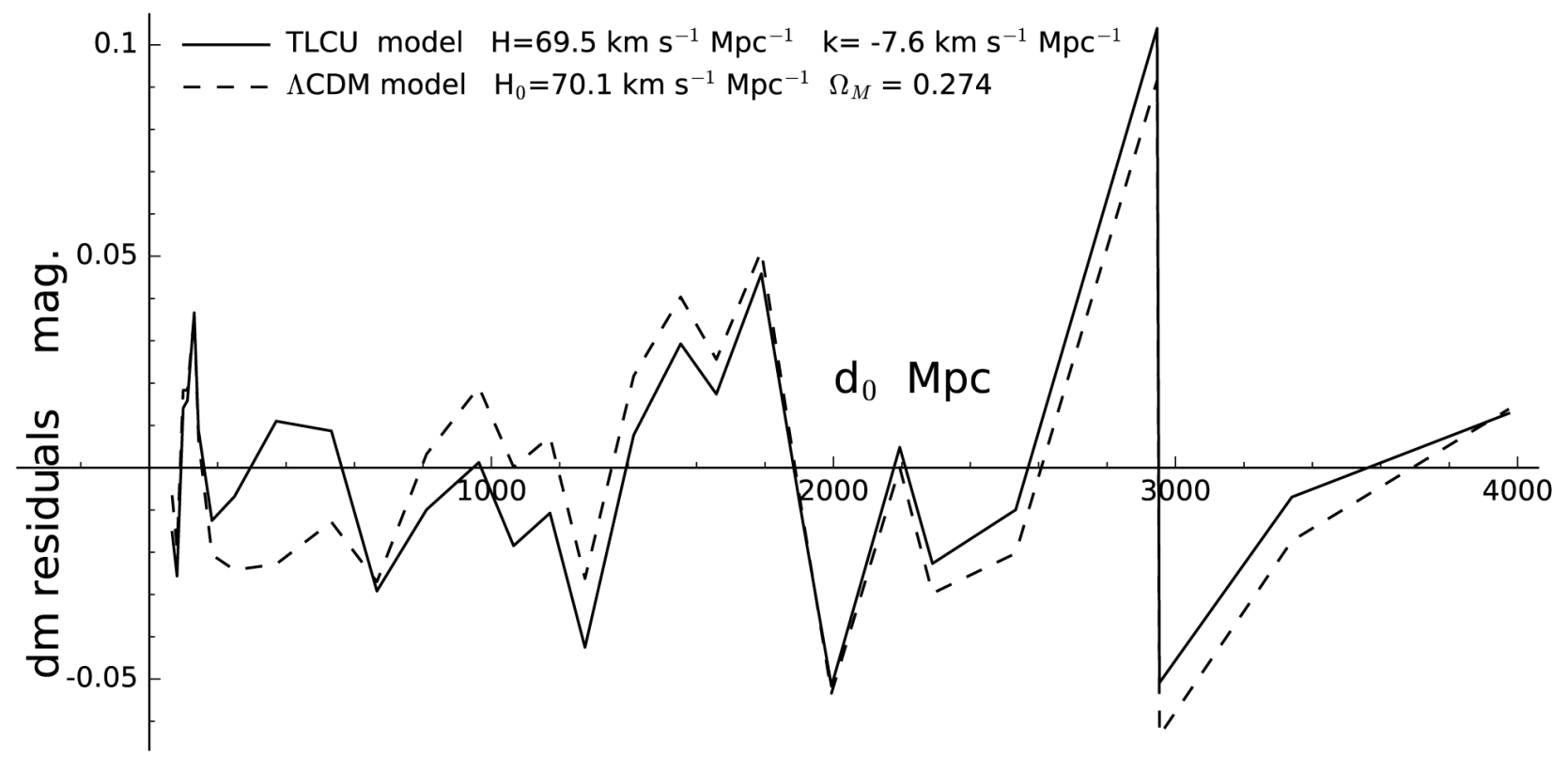

Figure 4. $d m$ residuals for $\Lambda C D M$ and TLCU models using binned data (see text). 
mechanism for the tired light process and a terrestrial experiment to test this mechanism are discussed in Appendix 2.

\section{Conclusion}

It is concluded that further experimental work on a possible photon energy loss process would be justified.

\section{Acknowledgements}

I am grateful to Graham P. Gerrard for help with computing and many stimulating discussions. I used TurboBasic for the calculations which were recoded in Python and the results checked by GPG using SageMathCloud (SMC). I am also grateful to the late Roger H. Beresford for a copy of the Nelder \& Mead minimisation routine coded in Basic. I used SMC to create the figures and SageTex to prepare the document.

\section{References}

[1] Friedman, A. (1922) Zeitschrift fur Physik, 10, 377-386 (translated (1999) General Relativity and Gravitation, 31, 1991-2000). https://doi.org/10.1023/A:1026751225741

[2] Hubble, E.A. (1929) Proceedings of the National Academy of Sciences, 15, 168-173. https://doi.org/10.1073/pnas.15.3.168

[3] Riess, A.G., et al. (1998) The Astronomical Journal, 116, 1009-1038. https://doi.org/10.1086/300499

[4] Perlmutter, S., et al. (1999) The Astrophysical Journal, 517, 565-586. https://doi.org/10.1086/307221

[5] Zwicky, F. (1929) Proceedings of the National Academy of Sciences, 15, 773-779. https://doi.org/10.1073/pnas.15.10.773

[6] Suzuki, N., et al. (2012) The Astrophysical Journal, 746, 85-108. https://doi.org/10.1088/0004-637X/746/1/85

[7] Kipper, A.Ya. (1974) Astrophysics, 10, 169-174. https://doi.org/10.1007/BF01006138

[8] MacMillan, W.D. (1932) Nature, 129, 93. https://doi.org/10.1038/129093a0

[9] Weinberg, S. (1972) Gravitation and Cosmology: Principles and Applications of the General Theory of Relativity. J. Wiley and Sons., New York.

[10] Reiss, A.G., et al. (2004) The Astrophysical Journal, 607, 665-687. https://doi.org/10.1086/383612

[11] Blondin, S., et al. (2008) The Astrophysical Journal, 682, 724-736. https://doi.org/10.1086/589568

[12] Wilkins, D.C. (1980) Physical Review D, 21, 2122-2136. https://doi.org/10.1103/PhysRevD.21.2122

[13] Hamster, H., Sullivan, A., Gordon, S., White, W. and Falcone, R.W. (1993) Physical Review Letters, 71, 2725-2728. https://doi.org/10.1103/PhysRevLett.71.2725 


\section{Appendix 1: Photon Travel Distance (d)}

The initial distance $\left(d_{0}\right)$ is the distance between emitter and observer at the moment a photon is emitted. In an expanding or contracting universe the path traversed by the photon will have expanded or contracted when it reaches the observer so that $d_{0} \neq d$ although $d_{0} \approx d$ for low values of $d_{0}$. The relationship between $\mathrm{d}$ and $d_{0}$ derived below is based on three assumptions: 1) the universe is flat [i.e. Euclidean], 2) the space velocity is much les than the speed of light, and 3) the space velocity is linearly proportional to distance

$$
\frac{\mathrm{d} x}{\mathrm{~d} t}=k \times x
$$

where $x$ is distance and $k$ is a constant. Integrating Equation (9) between $x=d_{0}$, $t=0$ and $x=d, t=t_{p}$ (where the photon travel time, $t_{p}=d / c$ )

$$
k \times \int_{o}^{t_{p}} \mathrm{~d} t=\int_{d_{o}}^{d}\left(\frac{1}{x}\right) \mathrm{d} x
$$

gives

$$
\frac{k \times d}{c}=\ln \left(\frac{d}{d_{o}}\right)
$$

Equation (11) is re-arranged as Equation (6) for use in Section 2.2.

\section{Appendix 2: Tired Light Experiment}

Mechanisms for the tired-light effect which involve photon/photon interactions or photon/baryon interactions would involve deflection and blurring of images which is not observed. However a possible mechanism which avoids the blurring problem is spontaneous photon decay [8] in which it is assumed that a primary photon decays producing secondary photons [12] all continuing to travel in the same direction. It is necessary that the frequency of these secondary photons would be considerably less than the frequency of the primary photon in order to avoid significant linebroadening .

Spontaneous decay of the primary radiation from the sun would produce secondary photons amounting to about $1.5 \times 10^{-12} \mathrm{Wm}^{-2}(H=70)$ at the earth. These tired light photons from the sun would need to be in the $\mathrm{GHz} / \mathrm{MHz}$ range or lower in order to avoid the line broadening effect and so would make a significant contribution to the observed quiet sun radio emission. Thus it may be that tired light secondary photons have already been observed and this raises the possibility of detecting the tired-light effect experimentally.

It is suggested here that the tired light radio emission which would be produced from a pulsed femtosecond optical laser could be detected in a terrestrial experiment. It would be necessary to conduct the laser beam through an evacuated tube in order to prevent the radio emission which would otherwise result [13] from ionization of the gas through which the laser beam travelled. 
Submit or recommend next manuscript to SCIRP and we will provide best service for you:

Accepting pre-submission inquiries through Email, Facebook, LinkedIn, Twitter, etc. A wide selection of journals (inclusive of 9 subjects, more than 200 journals)

Providing 24-hour high-quality service

User-friendly online submission system

Fair and swift peer-review system

Efficient typesetting and proofreading procedure

Display of the result of downloads and visits, as well as the number of cited articles Maximum dissemination of your research work

Submit your manuscript at: http://papersubmission.scirp.org/

Or contact jmp@scirp.org 